\title{
O ENFERMEIRO E A EDUCAÇÃo EM SAÚdE MENTAL NA ATENÇÃo PRIMÁRIA: REVISÃO INTEGRATIVA
}

Fernanda Ribeiro Sobral Claudinei José Gomes Campos²

Trata-se de revisão integrativa de literatura que analisou pesquisas sobre a participação dos enfermeiros nas ações de educação voltadas à saúde mental nos Centros de Saúde. Foram pesquisados, nas bases de dados BDENF, LILACS e SciELO, os artigos publicados entre 2001 e 2011. Encontraram-se oito artigos que mostraram dois temas principais: ações educativas em saúde voltadas à saúde mental, subdivididas em abordagem preventiva e promotora e dificuldades do enfermeiro na realização de ações educativas em saúde mental na atenção primária. Concluiu-se que houve poucos estudos sobre o tema, mostrando a necessidade de o Governo e as instituições formadoras de enfermagem incluírem, como prioridade política e curricular, as ações educativas em saúde mental na Estratégia Saúde da Família.

Descritores: Saúde Mental; Educação em Saúde; Atenção Primária à Saúde; Enfermagem.

\footnotetext{
${ }^{1} \mathrm{MSc}$.

${ }^{2}$ PhD, Professor Doutor, Universidade de Campinas, Campinas, SP, Brasil.
}

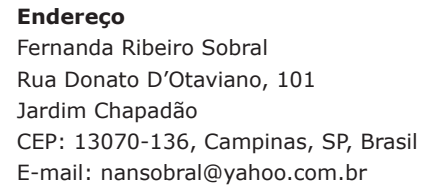




\section{NuRSES AND MENTAL HEALTH EDUCATION IN PRIMARY CARE: AN INTEGRATIVE}

\section{REVIEW}

This is a literature integrative review that has analyzed researches on the participation of nurses in education initiatives regarding mental health in Health Centers. We researched, in BDENF, LILACS and SciELO databases, articles published between 2001 and 2011. We found eight articles that showed two main themes: health education actions directed to mental health, subdivided into preventive and promoter approach; and difficulties of nurses in conducting educational activities in mental health in primary care. It was concluded that there were few studies on the subject, showing the need for government and nursing educational institutions to include as political and curricular priority educational activities on mental health in Family Health Strategy.

Descriptors: Mental Health; Health Education; Primary Health Care; Nursing.

\section{El ENFERMERO Y LA EDUCACIÓN EN SALUD MENTAL EN LA ATENCIÓN PRIMARIA: REVISIÓN INTEGRATIVA}

Revisión integrativa de literatura que analizó pesquisas sobre la participación de los enfermeros en las acciones de educación vueltas a la salud mental en los Centros de Salud. Fueron investigados, en las bases de dados BDENF, LILACS y SciELO, los artículos publicados entre 2001 y 2011. Se encontraron ocho artículos que mostraron dos temas principales: acciones educativas en salud vueltas a la salud mental, subdividida en abordaje preventivo y promotora; y dificultades del enfermero en la realización de acciones educativas en salud mental en la atención primaria. Se concluyó que hubo pocos estudios sobre el tema, mostrando la necesidad de Gobierno e instituciones formadoras de enfermería incluir como prioridad política y curricular las acciones educativas en salud mental en la Estrategia Salud de la Familia.

Descriptores: Salud Mental; Educación en Salud; Atención Primaria de Salud; Enfermería.

\section{Introdução}

O novo modelo de assistência psiquiátrica no Brasil priorizou, principalmente, a reinserção dos pacientes na sociedade e a ampliação da rede extra-hospitalar ${ }^{(1)}$. A Estratégia Saúde da Família (ESF) veio ao encontro das ideias propostas pela Reforma Psiquiátrica ${ }^{(2)}$, mas essa aumentou a responsabilidade da família, que foi obrigada a assumir uma participação ativa no processo de cuidado e na reinserção social dos pacientes adoecidos mentalmente. Porém, essa responsabilidade compartilhada de assistência com as equipes de saúde não forneceu a instrumentalização necessária aos familiares ${ }^{(3)}$.
Os serviços extra-hospitalares são insuficientes para atender à demanda de pacientes psiquiátricos e familiares, tanto em quantidade quanto em qualidade de cuidado, sendo difícil desenvolver atividades de prevenção dos fatores que facilitam a ocorrência de transtornos mentais ${ }^{(4)}$.

Nesse contexto, os Centros de Saúde (CS) são importantes componentes da rede em saúde mental em virtude dos vários atendimentos psiquiátricos que são realizados nesses locais ${ }^{(5)}$. Os CSs são essenciais na prevenção e promoção em saúde, sendo ambientes propícios para desenvolver ações educativas em saúde mental $^{(6)}$, pois estão próximos da comunidade e do 
paciente, permitindo à equipe maior conhecimento sobre a situação de saúde dos usuários ${ }^{(5,7)}$.

Com o objetivo de identificar as práticas em saúde mental do enfermeiro na ESF, encontrou-se que havia ausência de ações educativas em saúde mental ${ }^{(1)}$. Outro estudo identificou a assistência dada por enfermeiras da ESF diante dos casos psiquiátricos e não mostrou ações educativas em saúde mental ${ }^{(6)}$, sendo preciso investir em estratégias de promoção da saúde nessa área.

Apesar da baixa prioridade dada às ações educativas em saúde mental nos CSs, esses têm alto potencial para detectar fatores de risco para alguns tipos de doenças mentais, permitindo acompanhar e oferecer melhores possibilidades de controle e intervenção( ${ }^{(7)}$.

$\mathrm{Na}$ maioria dos países, os serviços de saúde mental têm baixa prioridade nas políticas de saúde pública. $\mathrm{O}$ investimento na área psiquiátrica concentra-se na terapia medicamentosa, cujo tratamento é longo e caro. Algumas doenças psiquiátricas podem ser prevenidas com ações menos onerosas e mais efetivas como atividades terapêuticas e ações educativas em saúde. Tais medidas podem reduzir custos em diversos contextos da $\operatorname{assistência(7).~}^{(7)}$.

A educação em saúde é a combinação de vários comportamentos humanos em saúde com várias experiências de aprendizagem e de ações educativas que permitam atitudes voluntárias, individuais ou coletivas, favoráveis à saúde ${ }^{(8)}$, para manter ou atingir um nível ideal de saúde, desde que seja adequado às necessidades de cada pessoa.

Hoje, a ação de educação em saúde é uma das principais estratégias no tratamento dos pacientes adoecidos mentalmente. A ação educativa é parte importante do trabalho do enfermeiro, cuja participação pode ser um diferencial no cuidado e fazer da ação educativa em saúde mental uma aliada do seu processo de trabalho, oferecendo melhor assistência ${ }^{(9)}$.

Este estudo objetivou analisar as produções científicas de periódicos nacionais que versam sobre as ações educativas em saúde voltadas à saúde mental, de pacientes e/ou familiares, no contexto dos CSs, e que tenham a participação de enfermeiros.

\section{Método}

Trata-se de revisão integrativa de literatura que sintetiza estudos já realizados, permitindo a inclusão simultânea de vários tipos de pesquisa, a reunião de dados da literatura teórica e empírica, proporcionando a compreensão completa e profunda dos conhecimentos de um fenômeno particular ${ }^{(10)}$. Assim, seguiram-se as principais etapas básicas da revisão, mostradas abaixo.

Identificação do problema de pesquisa - partiu-se da questão "há ações educativas em saúde voltadas à saúde mental nos CSs com a participação do enfermeiro?". Pressupõe-se que há escassez de ações e de artigos sobre o tema proposto.

Pesquisa bibliográfica - utilizou-se a seguinte sequência das bases de dados: Base de Dados de
Enfermagem (BDENF), Literatura Latino-Americana e do Caribe em Ciências da Saúde (LILACS) e consulta à biblioteca eletrônica de acesso aberto Scientific Eletronic Library Online (SciELO). A busca bibliográfica foi feita em janeiro de 2012.

Utilizaram-se termos presentes nos Descritores em Ciências da Saúde (DeCS) da Biblioteca Virtual em Saúde (BIREME): Educação em Saúde (ES), Enfermagem (Enf), Saúde da Família (SF), Saúde Mental (SM) e Atenção Primária à Saúde (APS). Fez-se o cruzamento entre os descritores: ES/Enf/SM, ES/Enf/SF, ES/SM, ES/ SF. Para buscar estudos não contemplados, realizou-se o cruzamento SM/APS. Outras possibilidades do termo APS foram testadas com os demais descritores citados, mas não revelaram novos textos significativos.

Os critérios de inclusão foram textos completos disponíveis on-line ou no acervo das bibliotecas da universidade, em periódicos nacionais, nos idiomas português, inglês ou espanhol, publicados entre janeiro de 2001 e dezembro de 2011. Os trabalhos deveriam abordar o tema: "ação educativa em saúde voltada à saúde mental no cenário dos CSs ou locais próximos a eles”. As ações educativas em saúde mental devem ser direcionadas tanto aos usuários, com ou sem adoecimento mental, quanto aos familiares. Essas ações de educação devem ser realizadas por enfermeiros, docentes-enfermeiros ou discentes da graduação ou da pós-graduação em enfermagem, ou ter a participação desses profissionais. Excluíram-se da amostra: teses, dissertações, editoriais, cartas ao editor e textos que não incluíam o tema pretendido.

Organização dos dados - organizaram-se formulários contendo informações sobre ano de publicação, título do artigo, autores, periódico, fonte de indexação, tipo de estudo, objetivos, resultados e conclusões, síntese dos textos selecionados e características das ações educativas como assunto abordado, público-alvo, local das ações e profissional envolvido.

Interpretação e análise dos dados - realizou-se a leitura exploratória de títulos e resumos, obtendo-se a amostra parcial, da qual se fizeram leituras flutuantes, seguidas da leitura atenta e criteriosa dos artigos na íntegra. A adaptação ao tema selecionou a amostra final.

Foi analisado o corpus dos artigos selecionados, utilizando-se dos seguintes passos: leitura flutuante dos textos e organização dos dados (pré-análise), exploração dos artigos, codificando conteúdos emergentes e representativos, categorização baseada na incidência do conteúdo e nas características dos estudos, tratamento dos resultados mais significativos, descrevendo-os e organizando-os sucinta e individualmente para facilitar a análise crítica, propuseram-se inferências, interpretações e a categorização dos dados ${ }^{(11)}$.

Apresentação e divulgação da revisão - os dados mais relevantes devem ser expostos e divulgados às comunidades científicas com interesse no saber produzido sobre o tema.

Apesar dos critérios usados, a revisão pode ter algumas limitações quanto aos descritores utilizados e aos cruzamentos realizados entre eles. Pode-se tanto encontrar 
muitos textos com temas diversos, dificultando a préseleção e permitindo descobrir parcela significativa de artigos, quanto ocultar textos importantes, restringindo a amostra final.

\section{Resultado}

Na busca (Tabela 1) encontraram-se 99 artigos dos quais, após a leitura de títulos e resumos, se excluíram 67
(68\%) textos. Dos 32 (32\%) artigos pré-selecionados, após a leitura na íntegra, eliminaram-se 24 (24\%), pois não se adequavam ao tema proposto. Portanto, no total, excluíramse $91(92 \%)$ artigos e a amostra final foi composta por 8 (8\%) publicações (Figura 1).

Tabela 1 - Distribuição dos artigos encontrados, excluídos e selecionados nas bases eletrônicas de dados. Brasil, 2001 a 2011

\begin{tabular}{lccccc}
\hline \multicolumn{1}{c}{ Dados } & Encontrados & Excluídos & Amostra parcial & $\begin{array}{c}\text { Excluídos da amostra } \\
\text { parcial }\end{array}$ & Amostra final $^{*}$ \\
\hline BDENF & 32 & 22 & 10 & 6 & $4(4 \%)$ \\
LILACS & 46 & 33 & 13 & 10 & $3(3 \%)$ \\
SciELO & 21 & 12 & 9 & 8 & $1(1 \%)$ \\
Total & 99 & $67(68 \%)$ & $32(32 \%)$ & $24(24 \%)$ & $8(8 \%)$ \\
\hline
\end{tabular}

*Dados numéricos em percentis arredondados

\begin{tabular}{|l|c|c|c|c|c|}
\hline \multicolumn{1}{|c|}{$\mathbf{n}$} & Ano & Título & Periódico & Fonte \\
\hline 1 & 2002 & $\begin{array}{c}\text { Ação educativa do enfermeiro direcionada para familiares de pacientes } \\
\text { com hanseníase: enfoque em saúde mental(12) }\end{array}$ & Rev. RENE & BDENF \\
\hline 2 & 2006 & $\begin{array}{c}\text { Educação e saúde mental na família: experiência com grupos } \\
\text { vivenciais(13) }\end{array}$ & $\begin{array}{c}\text { Texto \& contexto } \\
\text { enferm. }\end{array}$ & BDENF \\
\hline 3 & 2008 & $\begin{array}{c}\text { Saúde mental no contexto da atenção básica: potencialidades, } \\
\text { limitações, desafios do Programa Saúde da Família(14) }\end{array}$ & Rev. Eletr. Enf. & LILACS \\
\hline 4 & 2009 & $\begin{array}{c}\text { A terapia comunitária como estratégia de promoção à saúde mental: o } \\
\text { caminho para o empoderamento(15) }\end{array}$ & Rev. Eletr. Enf. & BDENF \\
\hline 5 & 2010 & $\begin{array}{c}\text { Grupo de ajuda a alcoolistas: a educação em saúde na Estratégia } \\
\text { Saúde da Família(16) }\end{array}$ & Cogitare enferm. & LILACS \\
\hline 6 & 2011 & $\begin{array}{r}\text { Saúde mental na atenção básica: uma abordagem convergente } \\
\text { assistencial(17) }\end{array}$ & $\begin{array}{c}\text { Rev. gauch. } \\
\text { enferm. }\end{array}$ & ES/Enf/SF \\
\hline 7 & 2011 & $\begin{array}{r}\text { Educação em saúde na Estratégia Saúde da Família: o significado e a } \\
\text { práxis dos enfermeiros(18) }\end{array}$ & Esc. Anna Nery & SM/APS \\
\hline 8 & 2011 & $\begin{array}{c}\text { O teatro em foco: estratégia lúdica para o trabalho educativo na saúde } \\
\text { da família(19) }\end{array}$ & Esc. Anna Nery & BDENF & ES/Enf/SF \\
\hline
\end{tabular}

Figura 1 - Estudos selecionados por ano de publicação, título, periódico, fonte de indexação e descritores, 2001 a 2011

Os tipos de estudos encontrados foram: duas $(25 \%)$ pesquisas bibliográficas, dois $(25 \%)$ relatos de experiência, quatro $(50 \%)$ estudos qualitativos (um descritivo, um exploratório, um descritivo-exploratório e uma pesquisa convergente assistencial - cada um com 12,5\%).

As ações educativas destinavam-se, em três $(37,5 \%)$ artigos, a pacientes da saúde mental, dois (25\%) a familiares; dois $(25 \%)$ a familiares e pacientes da saúde mental e um $(12,5 \%)$ era destinado a públicos diversificados, incluindo pacientes da saúde mental.

Os locais das atividades foram: três $(37,5 \%)$ somente em CS, três $(37,5 \%)$ em outros espaços cedidos às ações do CS (salões paroquiais de igrejas, clube municipal, escola da comunidade), dois (25\%) em CS e outros serviços de saúde e/ou de ensino.
Quanto ao principal profissional envolvido nas ações educativas, houve quatro (50\%) enfermeiros de CS, dois (25\%) eram docentes-enfermeiros trabalhando ora com enfermeiros discentes da pós-graduação (12,5\%) ora com um terapeuta ocupacional $(12,5 \%)$. Houve docenteenfermeiro e enfermeiros discentes da pós-graduação trabalhando juntos ou com um (12,5\%) enfermeiro de CS ou com um $(12,5 \%)$ terapeuta comunitário.

Dois artigos (25\%) relataram a utilização de abordagem de promoção de saúde mental; um (12,5\%) de prevenção, quatro $(50 \%)$ combinaram promoção e prevenção e, em um relato, a abordagem não estava clara.

A revisão agrupou os conteúdos dos artigos em dois temas: 1) ações educativas em saúde voltadas à saúde mental, subdividida em abordagem preventiva e promotora 
e 2) dificuldades na realização de ações educativas em saúde mental na ESF pelo enfermeiro.

\section{Discussão}

Ações educativas em saúde voltadas à saúde mental

Abordagem Preventiva

Prevenção é uma ação antecipada que anula, minimiza ou controla a evolução de uma doença ou seus fatores de riscos; reduz outros agravos associados, sua incidência e prevalência $^{(20-21)}$. Logo, ela pode ser feita antes ou depois da doença instalada ${ }^{(21)}$.

A prevenção tem três níveis: a primária diminui a incidência da doença e de seus fatores causais; aqui a prevenção precede a doença e se aplica a pessoas saudáveis ${ }^{(20)}$. Inclui ação de promoção da saúde, que pode ser uma intervenção biopsicossocial que promove o bem-estar e reduz a incidência da doença; e ação de proteção específica que exige a identificação do agente causador e do grupo vulnerável que está em risco ${ }^{(20-21)}$. As intervenções de enfermagem no nível primário incluem, principalmente, educação em saúde e apoio das redes sociais $^{(20)}$. Um estudo ${ }^{(13)}$ selecionado mostrou que uma família compreensiva e um ambiente escolar seguro são fatores de proteção que favorecem a prevenção de doenças.

A prevenção secundária se faz no indivíduo doente, buscando reduzir a incidência da doença. Suas intervenções são: diagnóstico, avaliação, tratamento precoce e imediato ${ }^{(20-21)}$. Entre os artigos selecionados, na abordagem preventiva, as ações realizaram-se sobre doenças instaladas e incluíram o desenvolvimento de estratégias para reduzir sofrimento mental/emocional, riscos e agravos das doenças mentais e danos em pacientes já adoecidos.

Já a terciária visa reduzir a gravidade da doença ou a incapacidade gerada, por meio da reabilitação ${ }^{(20-21)}$, que habilita o doente mental grave a atingir seu nível máximo possível de atuação, possibilitando que viva, aprenda e trabalhe em ambientes comunitários ${ }^{(20)}$.

A prevenção é uma ação ampla, multiprofissional e cabe, principalmente, aos profissionais de saúde "as decisões técnicas, as ações diretas e as ações educativas"(21). Assim, prevenção e educação em saúde estruturam-se mediante a divulgação de informações científicas e de recomendações normatizadas pelo Ministério da Saúde (MS), com ênfase nas mudanças de estilos de vida saudáveis ${ }^{(22)}$.

\section{Abordagem Promotora}

Promoção da saúde é o "processo de capacitação da comunidade para atuar na melhoria de sua qualidade de vida e saúde"(23). Ela não está restrita a aplicações técnicas e normativas, ao conhecimento sobre doenças e mecanismos de controle $^{(21)}$. Suas ações têm várias estratégias que permitem lidar com diversos determinantes de saúde e exercer maior controle sobre eles e suas condições de $\operatorname{vida}^{(21,24)}$, a fim de fazer escolhas favoráveis ou não à saúde.
Promove-se a saúde por meio de ações que ampliam a autonomia, o bem-estar biopsicossocial, o acesso aos serviços de saúde e sociais, favorecendo o empoderamento por meio de saberes e a participação nos movimentos de saúde, consolidando práticas intersetoriais ${ }^{(21,24)}$.

Diversos locais da comunidade podem oferecer opções para executar ações educativas ${ }^{(22)}$, cujos ambientes sejam favoráveis à promoção da saúde ${ }^{(25)}$. Percebeu-se, em alguns estudos ${ }^{(14,17-18)}$, que o local escolhido para fazer as ações educativas foi o CS, mas em outros ${ }^{(13,15-16)}$ buscaramse novos lugares, em razão da falta de espaço físico, da grande procura pelas atividades e do desejo de divulgar as ações em vários locais ${ }^{(19)}$.

Espaços coletivos construídos no CS permitem trocas dialógicas e horizontais, sendo ambientes de empoderamento que libertam e autonomizam as pessoas a tomarem decisões de saúde ${ }^{(17)}$. É positivo ampliar os ambientes da ação educativa: sair do cenário terapêutico tradicional e buscar espaços reais da vida das pessoas ${ }^{(14)}$. Porém, isso não isenta o CS da responsabilidade de desenvolver ações educativas com outros setores da comunidade.

Por outro lado, estudos mostraram que o ambiente familiar e o convívio social podem ser fatores de risco à criança em idade escolar ${ }^{(13)}$, às famílias que convivem com a doença mental ${ }^{(12,16)}$ e ao próprio paciente ${ }^{(15,17)}$; daí a importância de se valorizar o cuidado intrafamiliar, promovendo a saúde mental, prevenindo relações conflituosas, melhorando as relações afetivas, viabilizando o bem-estar e a qualidade de vida dos familiares e pacientes $^{(13)}$.

Já o trabalho com grupos terapêuticos é uma das principais estratégias da educação em saúde, para realizar intervenções em saúde mental ${ }^{(9,16)}$. Os grupos podem reduzir problemas, para a saúde pública e a sociedade, associados a diversas doenças mentais, dependência ao álcool e outras drogas ${ }^{(16)}$. É importante que os grupos tenham espaço nos CSs, pois esses têm ações relevantes ${ }^{(25)}$, baixo custo e maior possibilidade de acesso à população ${ }^{(15)}$.

$\mathrm{O}$ enfermeiro deve se preparar para atuar em grupos, pois essa atividade é muito utilizada na área da saúde mental ${ }^{(26)}$. O trabalho grupal realizado em alguns estudos da revisão ${ }^{(13-15,17)}$ é uma possibilidade para o enfermeiro prestar cuidado em saúde mental às famílias que convivem com pessoas em sofrimento psíquico. Os grupos, terapêutico e educativo, oferecem um cuidado integral, incluindo a família, cuja participação é essencial, pois é um apoio a si mesma e um auxílio ao desenvolvimento e cuidado do paciente ${ }^{(11,25)}$.

A promoção da saúde inclui estratégias terapêuticas e educativas, sendo que as primeiras encontradas nos artigos envolveram: trocas de experiências e reflexões, redução de estigmas e exclusões, aumento da autoestima, dos vínculos afetivos e sociais. Já as estratégias educativas caracterizaram-se por: melhorar a compreensão do processo saúde/doença mental, potencializar a autonomia e valorizar a cidadania, com participação ativa no cuidado $^{(25)}$

Ressalta-se que sete pesquisas apresentaram ações educativas voltadas à saúde mental com alguma abordagem 
promotora, sendo que em alguns estudos ela predominou como: o teatro, que foi uma alternativa pedagógica eficaz para os enfermeiros, pois ocorreu pela interação entre saber popular e científico para a aquisição de conceitos de saúde, recurso de lazer e espaço de convivência ${ }^{(19)}$, e a terapia comunitária, que foi um espaço de construção de vínculos e inserções sociais, alívio e prevenção do sofrimento, possibilitando a resiliência ${ }^{(15)}$.

Dificuldades do enfermeiro na realização de ações educativas em saúde mental

Deficiência na formação e capacitação do enfermeiro

Há deficiência na formação acadêmica e na prática profissional dos enfermeiros para aplicar ações de promoção à saúde, bem como de assistência integral, pois suas ideias esbarram na hegemonia do modelo biomédico curativista $^{(16)}$. Já as ações preventivas ainda predominam na prática, mas não avançam para uma concepção positiva de saúde que foca os recursos humanos e sociais para obter melhora na qualidade de vida ${ }^{(24)}$.

A capacitação em saúde mental dos profissionais da atenção básica enfatiza os agentes causadores das doenças mentais para detectar casos novos e interromper precocemente o adoecimento $^{(14)}$, portanto, mantém-se o foco na prevenção, enquanto faltam capacitações com foco na promoção da saúde ${ }^{(27)}$.

Deve-se rever a formação e a capacitação dos enfermeiros, pois muitos atendimentos focam as medicações, consultas individuais e outros, que são os conteúdos priorizados na maioria dos currículos de enfermagem. Embora os profissionais devessem estar habilitados para assumir o tratamento das doenças mentais comuns, nem sempre eles estão preparados para atuar em saúde mental na $\mathrm{ESF}^{(14)}$. Deve haver investimentos das instituições de ensino e de saúde para formar profissionais voltados à realização de grupos na assistência ${ }^{(26)}$.

Na prática, veem-se “despreparo e desmotivação dos profissionais de saúde para realizar ações centradas nas relações humanas, na produção de vínculo e na autonomia do usuário". Exemplo disso foi relatado em um dos estudos $^{(14)}$ quando houve desagrado da equipe ao se tentar abordar o tema da saúde mental no grupo de hipertensos na ESF.

Reconhece-se, porém, a complexidade em realizar esse trabalho, decorrente do estigma social da doença mental e que o próprio profissional possui ${ }^{(16)}$. Ademais, o contato com o sofrimento mental mobiliza vários sentimentos que sobrecarregam as pessoas. Isso deve ser considerado na capacitação/formação profissional, abrindo espaços para elaborar as dificuldades surgidas na relação com pacientes e familiares ${ }^{(14)}$.

É necessário e urgente despertar nos enfermeiros o interesse pela ação educativa, usar mecanismos de avaliação para identificar problemas na educação em saúde mental ${ }^{(17)}$, bem como oferecer suporte especializado e apoio matricial para fornecer aos profissionais "supervisão, estabelecer fluxos e referências resolutivas"(13).
Uso do modelo tradicional de ensino nas ações educativas

A equipe de saúde deve ser instrumentalizada segundo a necessidade do serviço para a promoção e a prevenção em saúde mental, utilizando, em suas ações educativas, propostas pedagógicas libertadoras ${ }^{(12,14)}$. O enfermeiro deve desenvolver trabalhos educativos diferenciados, com estratégias que facilitem a aprendizagem individual e coletiva $^{(19)}$, mas cujo conteúdo enfatize as necessidades da população com ações eficazes e eficientes.

As ações devem ser horizontais e dialógicas, entre profissionais e usuários do serviço, integrando saberes técnicos e populares ${ }^{(16,19)}$. Um artigo ${ }^{(19)}$ referiu que o teatro foi uma estratégia inovadora, que usou práticas lúdicas para humanizar a ação educativa, contemplar sentimentos e fortalecer a cidadania dos pacientes adoecidos mentalmente.

$\mathrm{Na}$ prática educativa dos profissionais do $\mathrm{CS}$, contudo, prevalece o modelo tradicional, o que pode indicar a complexidade do modelo de assistência integral e as dificuldades de utilizar novos métodos ${ }^{(18)}$. A prática tradicional que mais se destaca é a palestra, que apenas transmite informações, as quais são, comumente, normatizadas pelo $\mathrm{MS}^{(18,27)}$.

Tais atividades não visam o empoderamento das pessoas em conhecimentos de saúde, nem o exercício da cidadania, apenas atendem os interesses do MS e dos profissionais da ESF, que têm dificuldade em conciliar a alta demanda do serviço, as necessidades de saúde da população e as orientações que produzam impactos na saúde da comunidade ${ }^{(27)}$. Inclusive o usuário costuma procurar o CS pela possibilidade de estar doente e não para promover sua saúde ou prevenir a instalação de doenças. Por isso, a ação educativa também tende a voltar-se às pessoas doentes ou com riscos no estado de saúde ${ }^{(18)}$.

$\mathrm{Na}$ revisão, encontraram-se experiências de ações educativas em saúde mental inovadoras e realizadas com sistematização metodológica ${ }^{(15,17,19)}$, e outras com ações pontuais, eventuais e empíricas ${ }^{(13,18)}$, contribuindo para a descontinuidade da ação e o distanciamento do enfermeiro, pois esse tem sobrecarga de funções que predominam sobre suas práticas educativas em saúde voltadas à saúde mental e que não são padronizadas ${ }^{(18)}$.

Insuficiência e deficiência na rede de apoio intra e intersetorial

As articulações entre os serviços de saúde ainda se concentram em ações de referência e contrarreferência, com poucas construções de projetos $\operatorname{conjuntos}^{(27)}$ por intermédio de uma equipe especializada em saúde mental, como a do matriciamento. Isso ocorre porque a rede de saúde mental disponível para apoiar e restabelecer usuários e familiares nem sempre é suficiente, sendo, portanto, necessárias ações multidisciplinares ${ }^{(14)}$.

Quanto à intersetorialidade, a política de saúde mental se aproxima da política de promoção da saúde em relação à ampliação da autonomia e da cidadania dos sujeitos e na realização do cuidado integral. Ela é definida como: "relação entre uma ou várias partes do setor saúde com uma ou várias partes de outro setor", 
com o objetivo de "alcançar resultados de saúde mais eficientes"(28), articulando saberes diversos para formular e implementar ações de saúde, em que todos os setores se corresponsabilizam por sua efetivação.

Ações conjuntas em saúde poderiam motivar o enfermeiro a realizar ações educativas em saúde mental na ESF mais resolutivas ${ }^{(18)}$, mas na prática as ações intersetoriais são esporádicas e pouco efetivas ${ }^{(27)}$. $\mathrm{Na}$ revisão, houve estudos que valorizaram parcerias com escola pública ${ }^{(13)}$, igreja ${ }^{(16)}$, clube municipal ${ }^{(15)}$, creches, universidades e outros ${ }^{(19)}$.

É evidente que o setor da saúde tem dificuldade de atuar isoladamente e de implementar ações educativas efetivas, pois algumas parcerias dependem de locais diferenciados $^{(27)}$, principalmente na saúde mental, em relação à qual a rede de serviços comunitários disponível aos pacientes e familiares é insuficiente para atender suas diversas demandas ${ }^{(14)}$. Por isso, incentivam-se ações de saúde alternativas construídas na e com a comunidade ${ }^{(27)}$, corresponsabilizando-se com o setor público e os demais setores que não são da saúde ${ }^{(24)}$.

Assim, a maioria das ações são multissetoriais e não intersetoriais, pois essas são organizadas por um único setor para serem delegadas a outro. O planejamento de cuidado aos pacientes adoecidos mentalmente não apresenta uma discussão horizontal sobre as contribuições que cada setor pode dar. Esse é um sério desafio para concretizar a intersetorialidade que é erroneamente entendida pelos profissionais como uma responsabilidade individual de cada setor, fragmentando ainda mais o trabalho em rede ${ }^{(28)}$.

Falta de prioridade política para efetivar ações educativas em saúde mental na ESF

Outra dificuldade refere-se ao desenvolvimento e planejamento de políticas públicas que invistam nas ações educativas em saúde mental e estimulem o controle social $^{(18)}$. A participação efetiva da população é fundamental para a concretização da liberdade e da cidadania, ainda mais em relação ao paciente da saúde mental, quando visa a sua reinserção social ${ }^{(27)}$.

A atenção em saúde mental deveria ir além da lógica biologicista e promover convivência, autonomia, liberdade e cidadania ${ }^{(17)}$. Mas um estudo da revisão mostrou que dentre as várias ações educativas realizadas em diversos CSs, as orientações "em saúde" geralmente voltaram-se às doenças instaladas, como nos grupos para hipertensos, diabéticos, tabagistas e, inclusive, para o único grupo de saúde mental relatado na pesquisa ${ }^{(18)}$.

Isso decorre da influência do modelo biomédico, cujas intervenções focam a doença ${ }^{(18)}$. A superação desse modelo exige a reorientação do sistema de saúde, incluindo a organização, o financiamento dos serviços, as práticas e a formação dos profissionais ${ }^{(22)}$.

Atentando-se para essa afirmação: “a prevenção deve anteceder a ação dos especialistas em saúde, e no coletivo deve começar no nível das estruturas socioeconômicas e políticas"(20), faz-se necessário, para praticar a promoção, remodelar a assistência à saúde e mudar o processo de trabalho ${ }^{(18,27)}$, começando por alterar o foco na doença e visando o planejamento de ações para grupos vulneráveis a determinados fatores de risco ${ }^{(14)}$. Assim, a ação educativa também deveria ser utilizada para facilitar a reorganização dos serviços de saúde ${ }^{(18)}$.

Outro empecilho para efetivar as ações educativas em saúde mental nos CSs é a confusão entre práticas de prevenção e promoção ${ }^{(21)}$. Há, entre os profissionais, um conceito impreciso de promoção da saúde, focado na ausência de doenças. Daí a tendência em associar promoção às atividades de prevenção de doenças ${ }^{(27)}$, apontando seus fatores de risco (como nos grupos de hipertensão e diabetes) e propondo mudanças de comportamento (como exercícios, redução do fumo, álcool e outras drogas) ${ }^{(21)}$, muito preconizadas pelos programas do MS.

Tal obrigação ministerial intimida o profissional a prestar a assistência integral, fazendo-o agir como cumpridor de normas destinadas à cura e à prevenção de agravos, ações que não atendem as necessidades de toda a população e nem ao princípio básico do Sistema Único de Saúde (SUS): a universalidade. Os usuários que não se enquadram na oferta da ESF, como os pacientes em saúde mental, ficam excluídos da assistência dentro do seu território $^{(27)}$.

Quando há ações em saúde, inclusive educativa, que inclui grupos excluídos, percebe-se que o desenvolvimento e a realização dessas práticas não são uniformes, pois dependem do perfil e objetivos do profissional que atua na ESF ou de decisões políticas do gestor local ${ }^{(18)}$.

Também há falha nos registros dos sistemas de informação oficiais sobre as ações de saúde mental realizadas nos CSs. Isso prejudica a quantificação de dados e o conhecimento sobre os pacientes e suas famílias no território, impedindo planos de intervenção assertivos para a saúde mental e bloqueando os financiamentos para essa área na ESF. Inclusive, a falta de recursos financeiros impede a aquisição de recursos materiais e humanos para oferecer um serviço melhor ${ }^{(14)}$. Daí a importância de se construir parcerias com os setores públicos e privados para apoiar a realização das ações educativas em saúde mental $^{(19)}$.

\section{Considerações finais}

Apesar de a revisão apresentar uma participação significativa dos enfermeiros de CS nas ações educativas em saúde voltadas à saúde mental, houve poucos estudos que trataram sobre esse tema, mostrando a necessidade de as instâncias governamentais e instituições formadoras em saúde incluírem, entre as suas prioridades políticas e curriculares, a área da saúde mental, com ênfase nas ações educativas, tanto de promoção quanto de prevenção.

Vale ressaltar que se espera que os planejamentos de longo prazo na saúde mental e na saúde como um todo recebam investimentos profissionais, políticos e financeiros nas intervenções educativas individuais, familiares e comunitárias. Necessita-se, também, da organização e participação popular para mobilizar a população sobre a importância de ações promotoras e preventivas de qualidade, voltadas aos pacientes mentalmente adoecidos, além de exigências contumazes da sociedade e profissionais 
da saúde por decisões políticas incisivas no campo da saúde mental, principalmente, na atenção primária.

\section{Referências}

1. Souza AJF, Matias GN, Gomes KFA, Parente ACM. A saúde mental no Programa de Saúde da Família. Rev. Bras. Enferm. 2007;60(4):391-5.

2. Aires M, Roos CM, Gonçalves AVF, Schneider JF, Olschowsky A. Ações em saúde mental às famílias nos diferentes contextos de trabalho: revisão integrativa. Rev. gauch. enferm. 2010;31(3):567-74.

3. Cavalheri SC. Transformações do modelo assistencial em saúde mental e seu impacto na família. Rev. Bras. Enferm. 2010;63(1):51-7.

4. Souza AR, Moraes LMP, Barros MGT, Vieira NFC, Braga VAB. Estresse e ações de educação em saúde: contexto da promoção da saúde mental no trabalho. Rev. RENE. 2007;8(2):26-34.

5. Reinaldo AMS. Saúde mental na atenção básica como processo histórico de evolução da psiquiatria comunitária. Esc. Anna Nery. 2008;12(1):173-8.

6. Oliveira FB, Silva JCC, Silva VHF, Cartaxo CKA. O trabalho de enfermagem em saúde mental na estratégia de saúde da família. Rev. RENE. 2011;12(2):229-37.

7. World Health Organization. Promoting mental health: concepts, emerging evidence, practice: summary report. [Internet]. Geneva: WHO; 2005. [acesso 4 mar 2012]. Disponível em: http://www.who.int/mental_health/ evidence/MH_Promotion_Book.pdf

8. Candeias NMF. Conceitos de educação e de promoção em saúde: mudanças individuais e mudanças organizacionais. Rev. saude publica. 1997;31(2):209-13.

9. Zolnierek CD. Mental health policy and integrated care: global perspectives. J Psychiatr Ment Health Nurs. 2008;15(7):562-8.

10. Whittemore $\mathrm{R}$, Knafl $\mathrm{K}$. The integrative review: updated methodology. J Adv Nurs. 2005;52(5):546-53.

11. Bardin L. Análise de conteúdo. Lisboa: Editora 70; 2009.

12. Souza MM, Nobrega MML, Silva MIT. Ação educativa do enfermeiro direcionada para familiares de pacientes com hanseníase: enfoque em saúde mental. Rev. RENE. 2002;3(1):90-6.

13. Macêdo VC, Monteiro ARM. Educação e saúde mental na família: experiência como grupos vivenciais. Texto \& contexto enferm. 2006;15(2):222-30.

14. Munari DB, Melo TS, Pagotto V, Rocha BS, Soares CB, Medeiros M. Saúde Mental no contexto da atenção básica: potencialidades, limitações, desafios do Programa Saúde da Família. Rev. Eletr. Enf. 2008;10(3):784-95.

15. Ferreira Filha MO, Dias MD, Andrade FB, Lima EAR, Ribeiro FF, Silva MSS. A terapia comunitária como estratégia de promoção à saúde mental: o caminho para o empoderamento. Rev. Eletr. Enf. 2009;11(4):964-70.

16. Maciel MED, Pillon SC. Grupo de ajuda a alcoolistas: a educação em saúde na estratégia saúde da família. Cogitare enferm. 2010;15(3):552-5.

17. Antonacci MH, Pinho LB. Saúde mental na atenção básica: uma abordagem convergente assistencial. Rev. gauch. enferm. 2011;32(1):136-42.

18. Roecker S, Marcon SS. Educação em saúde na estratégia saúde da família: o significado e a práxis dos enfermeiros. Esc. Anna Nery. 2011;15(4):701-9. 19. Soares SM, Silva LB, Silva PAB. O teatro em foco: estratégia lúdica para o trabalho educativo na saúde da família. Esc. Anna Nery. 2011;15(4):818-24.

20. Stuart GW, Laraia MT. Contínuo do Tratamento Psiquiátrico. In: Stuart GW, Laraia MT, organizadores. Enfermagem Psiquiátrica. 4. ed. Rio de Janeiro: Reichmann \& Affonso Editores. 2002; p.112-32.

21. Rouquayrol AZ, Goldbaum M. Epidemiologia, história natural e prevenção de doenças. In: Rouquayrol AZ, Almeida Filho N, organizadores. Epidemiologia \& Saúde. 6. ed. Rio de Janeiro: MEDSI; 2003; p.17-35.

22. Czeresnia D. O conceito de saúde e a diferença entre prevenção e promoção. In: Czeresnia D, Freitas CM, organizadores. Promoção da saúde: conceitos, reflexões, tendências. Rio de Janeiro (RJ): Fundação Oswaldo Cruz. 2003; p.1-7.

23. Ministério da Saúde (BR). Secretaria de Políticas de Saúde. Projeto Promoção da Saúde. As Cartas da Promoção da Saúde. Brasília (DF): Ministério da Saúde; 2002.

24. Lopes MSV, Saraiva KRO, Fernandes AFC, Ximenes LB. Análise do conceito de promoção da saúde. Texto Contexto Enferm. 2010;19(3):461-8.

25. Benevides DS, Pinto AGA, Cavalcante CM, Jorge MSB. Cuidado em saúde mental por meio de grupos terapêuticos de um hospital-dia: perspectivas dos trabalhadores de saúde. Interface - Comunic., Saude, Educ. 2010;14(32):127-38.

26. Spadini LS, Souza MCBM. Conceito de grupo na percepção de enfermeiros na área de saúde mental e psiquiatria. SMAD, Rev. Eletrônica Saúde Mental Álcool Drog. 2011;7(3):133-8.

27. Horta NC, Sena RR, Silva MEO,Oliveira SR, Rezende VA.A prática das equipes de saúde da família: desafios para a promoção de saúde.Rev. bras. enferm. 2009;62(4):524-9. 28. Silva KL, Rodrigues AT. Ações intersetoriais para promoção da saúde na Estratégia Saúde da Família: experiências, desafios e possibilidades. Rev Bras Enferm. 2010; 63(5):762-9. 